\title{
Characterization of the Dynamics of an Essential Helix in the U1A Protein by Time-Resolved Fluorescence Measurements ${ }^{\dagger}$
}

\author{
Divina Anunciado,,$\S$ Michael Agumeh,, Bethany L. Kormos, $₫$ David L. Beveridge, \\ Joseph L. Knee, $*, *$ and Anne M. Baranger $*, \$, \S$ \\ Department of Chemistry and Molecular Biophysics Program, Wesleyan University, \\ Middletown, Connecticut 06459, and Department of Chemistry, University of Illinois at Urbana-Champaign, \\ Urbana, Illinois 61801
}

Received: August 28, 2007; In Final Form: November 29, 2007

\begin{abstract}
The RNA recognition motif (RRM), one of the most common RNA-binding domains, recognizes singlestranded RNA. A C-terminal helix that undergoes conformational changes upon binding is often an important contributor to RNA recognition. The N-terminal RRM of the U1A protein contains a C-terminal helix (helix C) that interacts with the RNA-binding surface of a $\beta$-sheet in the free protein (closed conformation), but is directed away from this $\beta$-sheet in the complex with RNA (open conformation). The dynamics of helix $\mathrm{C}$ in the free protein have been proposed to contribute to binding affinity and specificity. We report here a direct investigation of the dynamics of helix $\mathrm{C}$ in the free U1A protein on the nanosecond time scale using timeresolved fluorescence anisotropy. The results indicate that helix $\mathrm{C}$ is dynamic on a $2-3 \mathrm{~ns}$ time scale within a $20^{\circ}$ range of motion. Steady-state fluorescence experiments and molecular dynamics simulations suggest that the dynamical motion of helix $\mathrm{C}$ occurs within the closed conformation. Mutation of a residue on the $\beta$-sheet that contacts helix $\mathrm{C}$ in the closed conformation dramatically destabilizes the complex (Phe56Ala) and alters the steady-state fluorescence, but not the time-resolved fluorescence anisotropy, of a Trp in helix C. Mutation of Asp90 in the hinge region between helix $\mathrm{C}$ and the remainder of the protein to Ala or Gly subtly alters the dynamics of the U1A protein and destabilizes the complex. Together these results show that helix $\mathrm{C}$ maintains a dynamic closed conformation that is stable to these targeted protein modifications and does not equilibrate with the open conformation on the nanosecond time scale.
\end{abstract}

The RNA recognition motif (RRM) is the one of the most common RNA binding domains and is found in nearly all proteins involved in post-transcriptional gene expression. ${ }^{1-6}$ The $\beta \alpha \beta \beta \alpha \beta$ secondary structure of the RRM assembles into an RNA binding platform made up of a four-stranded antiparallel $\beta$-sheet, supported through an extensive hydrophobic core by two $\alpha$ helices. $^{7}$ The RRM is modulated in different proteins to recognize single-stranded RNAs with diverse sequences and in a variety of structural contexts. While specific amino acid sidechains are observed to contact RNA in structures of RRMRNA complexes, more subtle structural factors, including conformational changes and dynamic processes, may also be important contributors to binding affinity and specificity. $4,8,9$

A C-terminal helix that contributes to complex formation is often present in RRMs. This C-terminal helix plays diverse roles in different RRMs. For example, in the U1A protein, helix $\mathrm{C}$ changes position upon binding RNA. ${ }^{10,11}$ In contrast, in CstF64, helix $\mathrm{C}$ unfolds upon binding, ${ }^{12,13}$ while in the Sex lethal, $\mathrm{HuD}$, nucleolin, and Poly(A)-binding protein RRMs, unstructured C-terminal regions form helices upon binding RNA. ${ }^{14-18}$ Thus, the C-terminal amino acids of RRMs comprise a variable

\footnotetext{
† Part of the "Attila Szabo Festschrift".

* To whom correspondence should be addressed. (A.M.B.) E-mail: baranger@uiuc.edu. Address: Department of Chemistry, University of Illinois at Urbana-Champaign, Urbana, IL 61801. Phone: 217-244-7649. Fax: 217-244-8024. (J.L.K.) E-mail: jknee@wesleyan.edu. Address: Department of Chemistry, Wesleyan University, Middletown, CT 06459. Phone: 860-685-2727. Fax: 860-685-2211.

$\doteqdot$ Wesleyan University.

$\S$ University of Illinois at Urbana-Champaign.
}

region that may contribute to specific RNA recognition through participation in dynamical processes. In this paper, we report the characterization of the dynamics of the C-terminal helix of the U1A protein using time-resolved fluorescence anisotropy.

The U1A protein is a component of U1 snRNP, a subunit of the spliceosome. ${ }^{19,20} \mathrm{U} 1 \mathrm{~A}$ binds with high specificity to two related target sites, stem loop 2 of U1 snRNA (SL2 RNA) and an internal loop region in the pre-mRNA of U1A. ${ }^{21-23}$ Although U1A contains two RRMs, only the N-terminal RRM has been observed to bind RNA. ${ }^{24,25}$ The N-terminal RRM of U1A has been structurally characterized free and bound to SL2 RNA by X-ray crystallography and NMR spectroscopy. ${ }^{10,11,26}$ A comparison of the free and bound structures suggests that recognition requires extensive conformational changes in both the protein and RNA. A significant component of the protein conformational change occurs in the orientation of helix C (D90-K98) (Figure 1). In the NMR structure of U1A in the absence of RNA, helix $\mathrm{C}$ interacts with conserved residues in the RNA binding region on the surface of the $\beta$ sheet that is the primary site of RNA recognition, ${ }^{10}$ while in the complex helix $\mathrm{C}$ is positioned away from the $\beta$-sheet surface. ${ }^{11}$

The role of the conformational change of helix $\mathrm{C}$ in complex formation is not clear. For example, the protein may be captured by the RNA while helix $\mathrm{C}$ is transiently in the open conformation or the RNA may induce the observed conformational change upon binding. Energy calculations based on molecular dynamics (MD) simulations have suggested that the stabilities of the open and closed structures are comparable. ${ }^{27}$ The $\mathrm{X}$-ray 


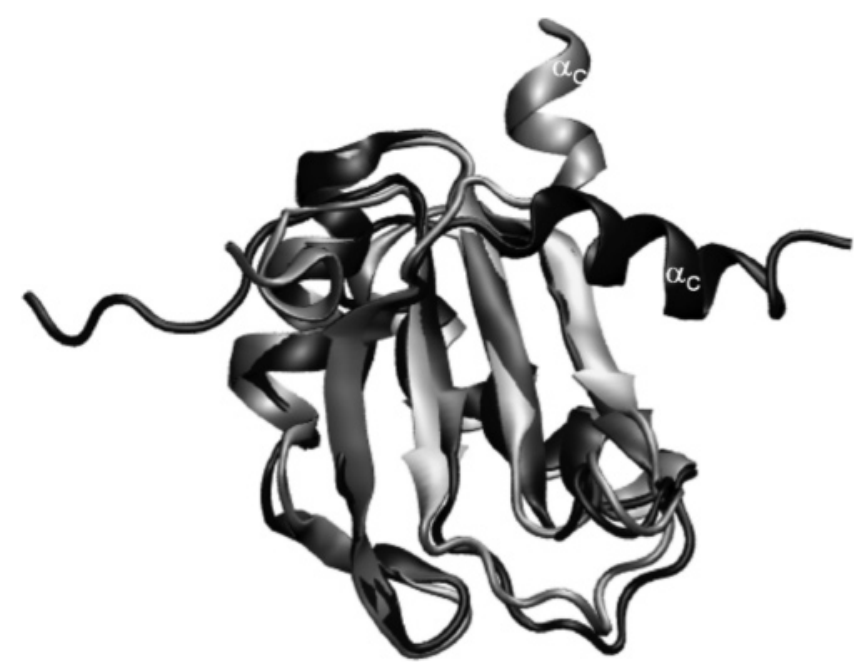

Figure 1. Diagram of the superposition of the free and bound conformations of the U1A protein. ${ }^{10,11}$ The darker diagram is the structure of the free form of U1A, while the lighter diagram is the structure of U1A in complex with SL2 RNA.

structure of a construct of the free protein with a relatively short helix $\mathrm{C}$ sequence showed that helix $\mathrm{C}$ is in the open conformation, supporting the result from MD studies that the open and closed structures are of similar stabilities. ${ }^{26}$ Fluorescence and NMR experiments and MD simulations have suggested that helix $\mathrm{C}$ retains significant flexibility in the free $\mathrm{U} 1 \mathrm{~A}$, although the time scale and range of dynamical motion has not been wellcharacterized. ${ }^{28-30}$ Mutational studies have shown that helix $\mathrm{C}$ contributes to binding affinity and specificity of the U1A protein for SL2 RNA and participates in cooperative networks of interactions with other residues involved in RNA binding. ${ }^{31,32}$ Studies using NMR, MD, and reorientational eigenmode dynamics techniques have suggested that these cooperative networks may originate in correlated dynamics involving helix C. ${ }^{30,33-35}$ Thus, the dynamical properties of helix $\mathrm{C}$ in the free protein are likely to make important cooperative contributions to RNA recognition by the U1A protein.

In this paper, we report time-resolved fluorescence anisotropy experiments of a U1A construct containing Trp in helix C to directly investigate the dynamics of helix $C$ on the picosecond to nanosecond time scale and to probe the influence of protein mutations on the dynamics of helix $\mathrm{C}$. The identification of the segmental dynamics of helix $\mathrm{C}$ in the U1A protein is supported by comparison to a U1A protein labeled with Trp in the stable $\beta$-sheet, rather than the $\mathrm{C}$-terminal helix. The data suggest the cone angle of motion of helix $\mathrm{C}$ to be $20^{\circ}$, which is similar to the cone angle predicted from molecular dynamics simulations performed on the U1A protein. ${ }^{27,36}$ Mutation of an amino acid on the surface of the $\beta$-sheet that contacts helix $\mathrm{C}$ or an amino acid in the hinge region between helix $\mathrm{C}$ and the remainder of the protein destabilizes the complex, but does not dramatically alter the dynamics of helix $\mathrm{C}$. Together these results suggest that helix $\mathrm{C}$ is not equilibrating between the closed and open form on the nanosecond time scale, but is undergoing a more limited degree of dynamical motion within the closed conformation that is relatively insensitive to mutation of residues that contact the helix or link the helix to the remainder of the protein.

\section{Materials and Methods}

General. Commercial reagents were used as received. DNA oligonucleotides and primers were purchased from QIAGEN
TABLE 1: Binding Affinities of Mutant U1A Proteins for SL2 RNA

\begin{tabular}{lcc}
\hline \multicolumn{1}{c}{ peptide } & $K_{\mathrm{D}}(\mathrm{M})^{a}$ & $\Delta G(\mathrm{kcal} / \mathrm{mol})^{b}$ \\
\hline wild type & $2( \pm 1) \times 10^{-10}$ & $-13.2 \pm 0.2$ \\
A95W & $3( \pm 1) \times 10^{-10}$ & $-12.9 \pm 0.3$ \\
K98W & $4( \pm 1) \times 10^{-10}$ & $-12.8 \pm 0.3$ \\
G99W & $4( \pm 2) \times 10^{-10}$ & $-12.8 \pm 0.4$ \\
F56A/K98W & $>6 \times 10^{-6}$ & \\
D90A/K98W & $3( \pm 2) \times 10^{-9}$ & $-11.7 \pm 0.3$ \\
D90G/K98W & $4( \pm 3) \times 10^{-8}$ & $-10.1 \pm 0.6$
\end{tabular}

${ }^{a} K_{\mathrm{D}}$ values are the average of at least three independent experiments. Standard deviations determined from the independent measurements are reported. ${ }^{b} \Delta G$ is the free energy of association of the complex.

and Integrated DNA Technologies (IDT). Sequencing was performed at UPenn, and mass spectrometry was performed at UIUC.

Protein Purification. An expression vector for the N-terminal RRM of the U1A protein, U1A101, was obtained from Nagai. ${ }^{37}$ Lys98Trp, Ala95Trp, Gly99Trp, Phe56Ala, Asp90Gly, and Asp90Ala mutations were introduced using standard Kunkel mutagenesis procedures. ${ }^{38}$ The proteins were expressed and purified as reported previously. ${ }^{39}$ The concentration of each protein was determined by amino acid analysis and the molecular weight by MALDI mass spectrometry.

Equilibrium Binding Assays. The equilibrium binding of SL2 RNA to the U1A protein was monitored by electrophoretic gel mobility shift assays. ${ }^{32} \mathrm{P}$-labeled SL2 RNA (0.025 nM) was incubated with competitor tRNA ( $1 \mathrm{mg} / \mathrm{mL})$ and varying amounts of U1A protein for $30 \mathrm{~min}$ at room-temperature in a buffer containing $10 \mathrm{mM}$ Tris- $\mathrm{HCl}, \mathrm{pH} 7.4,0.5 \%$ Triton $\mathrm{X}-100$, $1 \mathrm{mM}$ EDTA, and $250 \mathrm{mM} \mathrm{NaCl}$. After the addition of glycerol to a final concentration of $5 \%$, the bound and free RNA were separated using an $8 \%$ polyacrylamide gel (80:1 acrylamide/ bisacrylamide, $15 \mathrm{~cm} \times 40 \mathrm{~cm} \times 1.5 \mathrm{~mm})$ in $100 \mathrm{mM}$ Trisborate $\mathrm{pH} 8.3,1 \mathrm{mM}$ EDTA, $0.1 \%$ Triton $\mathrm{X}-100$ for $35 \mathrm{~min}$ at $350 \mathrm{~V}$. The temperature of the gel was maintained at $25^{\circ} \mathrm{C}$ by a circulating water bath. Gels were visualized on a molecular dynamics storm phosphorimager. Fraction RNA bound versus protein concentration was plotted and curves were fitted to the equation: fraction bound $=1 /\left(1+K_{\mathrm{d}} /[\mathrm{P}]_{\mathrm{T}}\right)$. All binding measurements were performed with a greater than 10 -fold excess of protein over RNA in each binding reaction used to determine the $K_{\mathrm{d}}$ so that $[\mathrm{P}]$ would be approximately equal to $[\mathrm{P}]_{\text {total }}$. Representative gel mobility shift assays and plots illustrating fraction RNA bound as a function of U1A concentration are shown in Figure 3. Dissociation constants and binding energies are listed in Table 1.

Circular Dichroism Spectroscopy. CD spectra were recorded on a Jasco J-810 CD spectrometer equipped with a Peltier temperature controller and Spectra Manager program. CD spectral scans were recorded from 190 to $300 \mathrm{~nm}$ in $0.1 \mathrm{~nm}$ increments with a scanning speed of $50 \mathrm{~nm} / \mathrm{min}$ and a $8 \mathrm{~s}$ response time using rectangular cells with a $0.2 \mathrm{~cm}$ path length.

Steady-State Fluorescence Measurements. Steady-state fluorescence experiments were carried out on a FluoroMax-3 Spex spectrofluorometer from Jobin Yvon Inc. Experiments were performed at $25{ }^{\circ} \mathrm{C}$ in $50 \mathrm{mM} \mathrm{KCl}, 10 \mathrm{mM} \mathrm{K}_{2} \mathrm{PO}_{4}$, pH 7.4. Excitation and emission slits were set to 4 and $8 \mathrm{~nm}$, respectively.

Lifetime Measurements and Time-Resolved Anisotropy. Fluorescence lifetimes and anisotropy decay were measured using time-correlated single photon counting (TCSPC). The excitation laser system consists of a cw mode-locked Nd:YAG laser (Coherent Antares) which was frequency doubled to 532 
TABLE 2: Fluorescence Decay Data

\begin{tabular}{|c|c|c|c|c|c|c|}
\hline & K98W & G99W & F56W & $\begin{array}{c}\text { F56A } \\
\text { K98W }\end{array}$ & $\begin{array}{l}\text { D90A } \\
\text { K98W }\end{array}$ & $\begin{array}{l}\text { D90G } \\
\text { K98W }\end{array}$ \\
\hline$\chi^{2}$ & $1.23 \pm 0.7$ & $1.09 \pm 0.2$ & $1.27 \pm 0.08$ & $1.23 \pm 0.17$ & $1.29 \pm 0.20$ & $1.33 \pm 0.07$ \\
\hline$\alpha_{1}$ & $0.28 \pm 0.03$ & $0.27 \pm 0.04$ & $0.17 \pm 0.02$ & $0.25 \pm 0.02$ & $0.37 \pm 0.10$ & $0.39 \pm 0.04$ \\
\hline$\tau_{1}(\mathrm{~ns})$ & $0.37 \pm 0.05$ & $0.40 \pm 0.05$ & $0.74 \pm 0.32$ & $0.46 \pm 0.08$ & $0.34 \pm 0.03$ & $0.40 \pm 0.06$ \\
\hline$\alpha_{2}$ & $0.43 \pm 0.02$ & $0.47 \pm 0.04$ & $0.53 \pm 0.07$ & $0.47 \pm 0.07$ & $0.39 \pm 0.07$ & $0.37 \pm 0.03$ \\
\hline$\tau_{2}(\mathrm{~ns})$ & $2.94 \pm 0.28$ & $2.59 \pm 0.3$ & $3.16 \pm 0.16$ & $2.87 \pm 0.33$ & $2.69 \pm 0.12$ & $2.71 \pm 0.16$ \\
\hline$\alpha_{3}$ & $0.29 \pm 0.04$ & $0.26 \pm 0.03$ & $0.31 \pm 0.06$ & $0.28 \pm 0.08$ & $0.24 \pm 0.04$ & $0.24 \pm 0.02$ \\
\hline$\tau_{3}(\mathrm{~ns})$ & $6.39 \pm 0.22$ & $5.66 \pm 0.4$ & $6.00 \pm 0.17$ & $6.32 \pm 0.47$ & $6.26 \pm 0.36$ & $7.00 \pm 0.16$ \\
\hline
\end{tabular}

nm and used to synchronously pump a cavity dumped dye laser. The dye laser was operated at $3.8 \mathrm{MHz}$ and produced a total power of approximately $100 \mathrm{~mW}$ at $610 \mathrm{~nm}$ with a pulse width of 10 psec. The dye laser was frequency doubled in KDP to produce the $305 \mathrm{~nm}$ UV used for excitation with an average power typically less than $1 \mathrm{~mW}$. The UV passed through a vertically oriented polarizer to ensure complete polarization and then was directed to the sample cuvette. Fluorescence was collected and collimated at right angles with f/1 optics and then focused with an f/7 lens onto the entrance slit of a 0.1 meter monochromator (ISA, Inc., model DH-10). A large aperture sheet polarizer was placed in the detection path. The orientation of the analyzing polarizer was computer controlled. After the analyzing polarizer, but before the entrance to the monochromator, a quartz depolarizer (Karl Lambrect Corp.) was placed to minimize any polarization bias effects in the monochromator and detector. The detector was a microchannel plate photomultiplier (Hamamatsu Inc. R1564U-06). The signal was collected in the single photon counting mode and capture by a PC-based multichannel analyzer (FAST ComTec, GmbH). Typical fluorescence decay curves consisted of 8192 time channels over a total range of $50 \mathrm{nsec}$ with the counts in the peak channel of the parallel component accumulated to a total of 20000 . A typical experiment consisted of acquiring data with the analyzing polarizer alternatively oriented parallel, magic angle, and perpendicular. Each orientation was sampled for $20 \mathrm{~s}$ and the process repeated until the counts in the peak channel for the parallel signal was 20000 counts. A number of emission wavelengths were measured but no systematic difference was noted with wavelength so the majority of the data was taken at $380 \mathrm{~nm}$. The system response function was acquired periodically by measuring scattered light from a colloidal suspension. The typical response function had a full width at half-maximum (fwhm) of approximately 80 ps and it was used in the fitting process to deconvolute the molecular response from the measured experimental decay curves.

The fluorescence decay curves were fit using the commercially available program Globals Unlimited. ${ }^{40}$ It was found that a three exponential decay model was required to adequately fit the magic angle tryptophan fluorescence decay data, which is consistent with the previously reported measurements on F56W U1A by Hall et al. ${ }^{28}$ The criteria for a good fit were the $\chi^{2}$ values and a visual inspection of the residual. Using a twocomponent, or less, exponential fit led to significantly worse values of $\chi^{2}$ and resulted in the residual having a noticeable systematic variation. Typical values of $\chi^{2}$ ranged from $1.03-$ 1.30 (Table 2).

The time dependent anisotropy was fit using the parallel and perpendicular data sets. The time dependent anisotropy is defined as follows:

$$
r(t)=\frac{I_{\|}(t)-I_{\perp}(t)}{I_{\|}(t)+2 I_{\perp}(t)}
$$

The experimentally measured anisotropy decay, $r(t)$, is typically modeled as the sum of one or more exponential decay terms.

$$
r(t)=r_{0} \sum_{i} \beta_{i} \mathrm{e}^{-t / \theta_{i}}
$$

Where $r_{0}$ is the limiting anisotropy ( 0.4 for parallel absorption and emission transition dipoles), $i$ is the index for the number of exponentials contributing to the observed signal ( 2 or 3 for this work), $\beta_{i}$ are the weighting factors for each contributing exponential, and $\theta_{i}$ is the rotational correlation time for component $i$. The sum of the coefficients, $\beta_{i}$, is normalized to one, $\sum_{i} \beta_{i}=1$.

The molecular motions associated with loss of anisotropy are not directly given by the above model but are somewhat more complicated because the chromophore is possibly subject to several dynamical processes simultaneously which can lead to a loss of anisotropy. For the tryptophan considered in this work we identify three types of motion that are possible (although not always observed for each protein). The fastest motion is the local tryptophan motion due to rotation about its $\mathrm{C}_{\alpha}-\mathrm{C}_{\beta}$ and $\mathrm{C}_{\beta}-\mathrm{C}_{\gamma}$ bonds, to which we will ascribe a rotational correlation time of $\theta_{\mathrm{L}}$ (local motion). The intermediate timescale motion is the segmental motion due to fluctuations in the local structure or internal motions of the protein. This intermediate time scale will be designated $\theta_{\mathrm{S}}$ (segmental motion). The slowest motion will be the overall rotation or tumbling of the molecule, which is designated $\theta_{\mathrm{R}}$ (rotational motion). The overall time dependent anisotropy in terms of these molecular motions can then be written as follows:

$$
r(t)=r_{0}\left[\left(\beta_{1} \mathrm{e}^{-\left(t / \theta_{\mathrm{L}}\right)}+\beta_{2}\right) \mathrm{e}^{-\left(t / \theta_{\mathrm{S}}\right)}+\beta 3\right] \mathrm{e}^{-\left(t / \theta_{\mathrm{R}}\right)}
$$

This can be rearranged to

$r(t)=r_{0}\left(\beta_{1} \mathrm{e}^{-t\left(1 / \theta_{\mathrm{L}}+1 / \theta_{\mathrm{S}}+1 / \theta_{\mathrm{R}}\right)}+\beta_{2} \mathrm{e}^{-t\left(1 / \theta_{\mathrm{S}}+1 / \theta_{\mathrm{R}}\right)}+\beta_{3} \mathrm{e}^{t / \theta_{\mathrm{R}}}\right)$

With reference to eq 1 :

$$
\frac{1}{\theta_{1}}=\frac{1}{\theta_{\mathrm{L}}}+\frac{1}{\theta_{\mathrm{S}}}+\frac{1}{\theta_{\mathrm{R}}}, \frac{1}{\theta_{2}}=\frac{1}{\theta_{\mathrm{S}}}+\frac{1}{\theta_{\mathrm{R}}} \text { and } \frac{1}{\theta_{3}}=\frac{1}{\theta_{\mathrm{R}}}
$$

From the above expressions we can determine $\theta_{\mathrm{L}}, \theta_{\mathrm{S}}$, and $\theta_{\mathrm{R}}$ in terms of the experimentally measured correlation times.

$$
\begin{gathered}
\theta_{\mathrm{R}}=\theta_{3} \\
\theta_{\mathrm{S}}=\frac{\theta_{3} \theta_{2}}{\theta_{3}-\theta_{2}} \\
\theta_{\mathrm{L}}=\frac{\theta_{1} \theta_{2}}{\theta_{2}-\theta_{1}}
\end{gathered}
$$

In one case only a two component decay is observed and this is ascribed to the absence of any appreciable segmental 
TABLE 3: Data from Time-Resolved Anisotropy Measurements ${ }^{a}$

\begin{tabular}{lccclll}
\hline & K98W & G99W & F56W & F56A/K98W & D90A/K98W & D90G/K98W \\
\hline$r_{0}$ & $0.29 \pm 0.03$ & $0.27 \pm 0.02$ & $0.31 \pm 0.06$ & $0.29 \pm 0.02$ & $0.33 \pm 0.05$ & $0.29 \pm 0.04$ \\
$\beta_{1}$ & $0.29 \pm 0.03$ & $0.25 \pm 0.03$ & $0.18 \pm 0.12$ & $0.27 \pm 0.02$ & $0.38 \pm 0.11$ & $0.43 \pm 0.04$ \\
$\theta_{\mathrm{L}}(\mathrm{ns})$ & $0.09 \pm 0.03$ & $0.18 \pm 0.03$ & $0.12 \pm .08$ & $0.11 \pm 0.03$ & $0.08 \pm 0.07$ & $0.10 \pm 0.05$ \\
$\beta_{2}$ & $0.22 \pm 0.05$ & $0.32 \pm 0.04$ & & $0.24 \pm 0.05$ & $0.13 \pm 0.03$ & $0.20 \pm 0.05$ \\
$\theta_{\mathrm{S}}(\mathrm{ns})$ & $2.58 \pm 0.44$ & $2.27 \pm 0.30$ & & $2.57 \pm 0.60$ & $1.96 \pm 0.88$ & $2.22 \pm 0 / 67$ \\
$\beta_{3}$ & $0.49 \pm 0.04$ & $0.43 \pm 0.05$ & $0.82 \pm 0.16$ & $0.49 \pm 0.10$ & $0.49 \pm 0.05$ & $0.37 \pm 0.08$ \\
$\theta_{\mathrm{R}}(\mathrm{ns})$ & $7.56 \pm 0.46$ & $6.63 \pm 0.76$ & $7.06 \pm 0.57$ & $7.22 \pm 0.31$ & $6.77 \pm 0.28$ & $6.53 \pm 0.55$
\end{tabular}

${ }^{a} \mathrm{r}_{0}, \beta_{1}, \theta_{\mathrm{L}}, \beta_{2}, \theta_{\mathrm{S}}, \beta_{3}$, and $\theta_{\mathrm{R}}$ are defined in the experimental section.

TABLE 4: Comparison of 2-Component and 3-Component Fitting of the Anisotropy Decay Data from the Phe56Trp, Lys98Trp, and Phe56Ala/Lys98trp U1A Proteins ${ }^{a}$

\begin{tabular}{lccccccc}
\hline \multicolumn{1}{c}{ protein } & $\beta_{\mathbf{1}}$ & $\theta_{\mathbf{L}}(\mathrm{ns})$ & $\beta_{\mathbf{2}}$ & $\theta_{\mathbf{S}}(\mathrm{ns})$ & $\beta_{\mathbf{3}}$ & $\theta_{\mathbf{R}}(\mathrm{ns})$ & $\chi^{\mathbf{2}}$ \\
\hline F56W & 0.30 & 0.07 & & & 0.70 & 7.06 & 1.25 \\
K98W & 0.30 & 0.11 & & & 0.70 & 4.51 & 1.19 \\
K98W & 0.28 & 0.06 & 0.24 & 2.52 & 0.48 & 6.98 & 1.17 \\
F56A/K98W & 0.27 & 0.20 & & & 0.73 & 4.40 & 1.23 \\
F56A/K98W & 0.23 & 0.14 & 0.32 & 2.90 & 0.45 & 7.07 & 1.21
\end{tabular}

${ }^{a}$ The specific values in this table differ slightly from the values in Table 3 because these values are from single data sets, whereas Table 3 presents the average of a number of data sets.

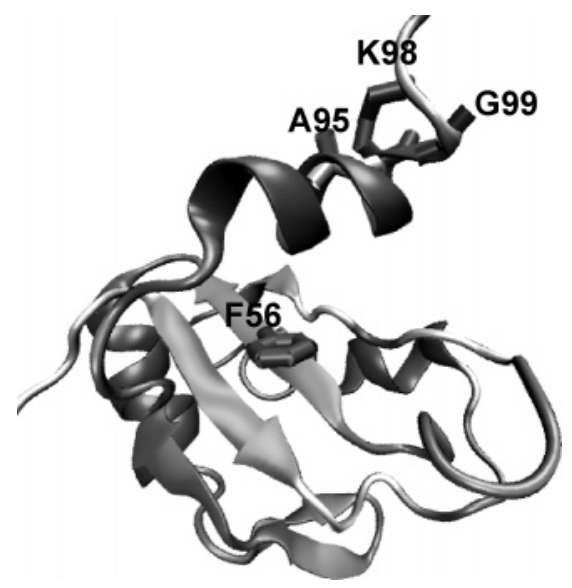

Figure 2. Diagram of the structure of the free U1A protein showing the positions of incorporation of Trp into helix C: Ala95, Lys98, Gly99, and Phe56.

motion. The data from the time-resolved anisotropy measurements are listed in Table 3.

The errors reported in Tables 3 and 4 are the standard deviations of multiple data sets with 3 to 10 individual experiments. In almost all cases these errors bars exceed the statistical errors reported by the fitting program for each individual fit but were typically consistent in magnitude.

Solvent Accessibility Calculations. Estimations of the solvent-accessible surface area (SASA) on a per-residue basis were computed from a $5 \mathrm{~ns}$ MD simulation of wild type U1A protein ${ }^{36}$ by recursively approximating a sphere around an atom starting from an icosahedra using the ICOSA keyword in Amber8. ${ }^{41,42}$ Snapshots for analysis were collected at $20 \mathrm{ps}$ intervals over the stable final $3 \mathrm{~ns}$ of the simulation for a total of 150 snapshots. The SASA values were normalized by the extended Ala-X-Ala SASA values for each residue, $\mathrm{X} \cdot{ }^{43}$

\section{Results}

Selection of Positions To Incorporate Trp in Helix C of the U1A Protein. The first objective was to establish if there was a measurable anisotropic fluorescence decay associated with the motion of helix $\mathrm{C}$ of U1A. The fluorescent label Trp was introduced into helix $\mathrm{C}$ at three positions, Ala95Trp, Lys98Trp, and Gly99Trp, which were chosen to minimize disruption to the free or bound structures, while being positioned within or adjacent to the well-structured part of helix C (Figure 2). The anisotropy decay signals from different labeled proteins were compared to distinguish between contributions from the motions of the indole side chain, which may differ between the three positions, and the motion of helix $\mathrm{C}$, which should remain constant in the three labeled proteins. The binding of Lys98Gln U1A protein for SL2 RNA has been found previously to be 10-50-fold weaker than that of the wild-type protein, ${ }^{37}$ while deletion of residues 98-101 reduced binding affinity only 2-fold. ${ }^{32}$ The binding affinities of U1A proteins containing mutations of Ala95 or Gly99 have not been reported previously.

Characterization of U1A Proteins Containing Trp Labels. Circular dichroism (CD) spectra of the wild type and Trp-labeled U1A proteins (amino acids 2-102) were similar and stable until $80{ }^{\circ} \mathrm{C}$, suggesting that the three Trp-labeled proteins are stably folded with structures that are similar to the wild-type protein. Gel mobility shift assays were performed to investigate the effect of the Trp substitutions on the ability of the proteins to bind RNA. The binding affinities of the labeled proteins were within error of that of the wild-type U1A Lys98Trp (Figure 3 and Table 1). Thus, the $\mathrm{CD}$ and binding experiments suggest that the Ala95Trp, Lys98Trp, and Gly99Trp U1A proteins are similar to the wild-type protein. We performed most of the experiments reported here with U1A proteins labeled with Lys98Trp because the Trp label is one residue closer to the main body of the protein than in Gly99Trp, and preliminary CD experiments on peptide models of helix $\mathrm{C}$ suggested that the helix containing the Ala95Trp substitution may be less structured than helices containing Lys98Trp or Gly99Trp substitutions.
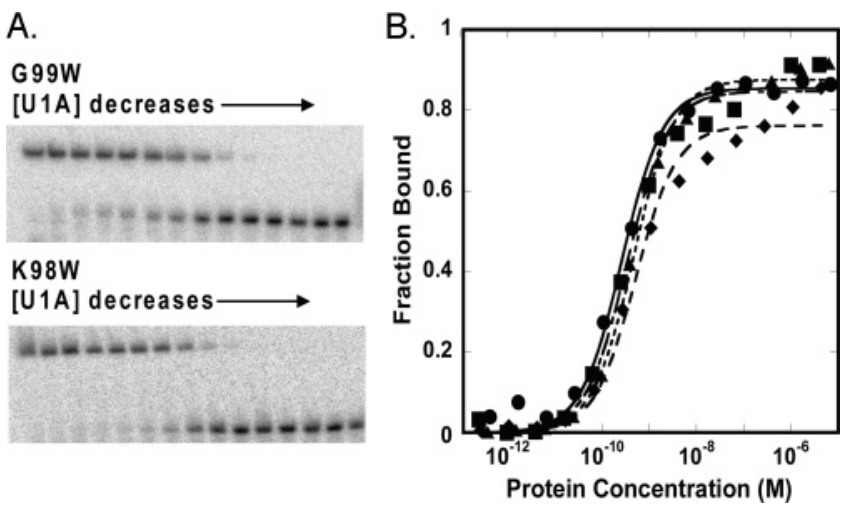

Figure 3. (A) Examples of gel mobility shift analyses of the Lys98Trp and Gly99Trp U1A proteins binding to SL2 RNA. In each gel, the slower moving band is the complex and the faster moving band is the free RNA. The highest protein concentration was $6.5 \mu \mathrm{M}$ for Lys $98 \mathrm{Trp}$ and $6 \mu \mathrm{M}$ for Gly99Trp proteins, and a 0.25 serial dilution of the protein was performed in each case. (B) Plots illustrating the fraction of SL2 RNA bound as a function of U1A protein concentration: wild type

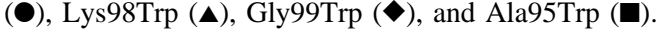




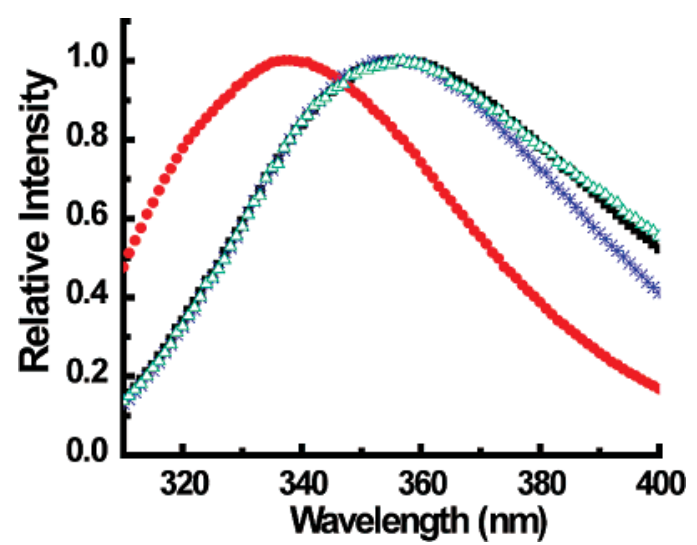

Figure 4. Steady-state fluorescence spectra of Lys98Trp (light blue), Gly99Trp (blue), Ala95Trp (black), and Phe56Trp (red) proteins (1 $\mu \mathrm{M})$ using a $\lambda_{\text {exc }}$ of $295 \mathrm{~nm}$ at $25^{\circ} \mathrm{C}$ in $50 \mathrm{mM} \mathrm{KCl}$ and $10 \mathrm{mM} \mathrm{K}_{2}-$ $\mathrm{PO}_{4}(\mathrm{pH}$ 7.4).

Steady-State Fluorescence of U1A Proteins Containing Trp Labels. The steady-state fluorescence spectra of the Lys98Trp, Gly99Trp, and Ala95Trp proteins are similar with emission maxima of $356 \mathrm{~nm}$ when excited at $295 \mathrm{~nm}$ (Figure 4). The emission maxima are relatively independent of excitation wavelength (data not shown). A value of $356 \mathrm{~nm}$ for the emission maxima suggests significant solvent exposure of the Trp side chains, which is consistent with the location of Trp in helix $\mathrm{C}$ at positions that do not contact the surface of the $\beta$-sheet in the free form of the protein (Figure 2). ${ }^{44}$ As a control for the fluorescence experiments, we investigated a U1A mutant containing Trp in the $\beta$-sheet, rather than in helix C, Phe56Trp (Figure 2). We previously have found that the Phe56Trp mutation does not alter the stability of the complex within experimental error. ${ }^{39}$ The fluorescence properties of this protein have been reported previously by Hall and co-workers. ${ }^{28}$ The emission maximum of the Phe56Trp U1A protein is significantly blue-shifted compared to the proteins containing Trp in helix C (Figure 4). In addition, the emission maximum varies with excitation wavelength (data not shown). These results are similar to those reported previously and indicate that the Trp in Phe56Trp is less solvent accessible than those in the Ala95Trp, Lys98Trp, or Gly99Trp proteins, presumably in part due to interactions between the surface of the $\beta$-sheet and helix C.

Time-Resolved Fluorescence Anisotropy Measurements. Time-resolved fluorescence anisotropy experiments were performed on the U1A proteins labeled with Trp in helix $\mathrm{C}$ to probe helix $\mathrm{C}$ motion (Tables 3 and 4 and Figure 5). Time-resolved fluorescence anisotropy is a well-established method to measure dynamics on the picosecond to nanosecond time scales in proteins and protein-nucleic acid complexes. ${ }^{45-49}$ For example, methods that are similar to the ones reported here have been used to characterize segmental motion of $\alpha$-helix $N$ in DNA polymerase $\beta .{ }^{47} \mathrm{We}$ performed experiments with both Lys98Trp and Gly99Trp U1A proteins, because we expected that comparison of the data from proteins labeled at two positions in helix $\mathrm{C}$ would contribute to a reliable assignment of the segmental dynamics of helix C. In addition, several anisotropy experiments were run on the Ala95Trp mutant and the results were similar to the Lys98Trp and Gly99Trp mutants, although not reported in detail since they were run at an earlier time using slightly different experimental methodologies. As a control experiment for the anisotropic decay associated with helix $\mathrm{C}$ motion, we investigated the U1A mutant containing Trp in the $\beta$-sheet, rather than in helix $\mathrm{C}$, Phe56Trp. The time-resolved fluorescence of the Phe56Trp U1A protein has been character-

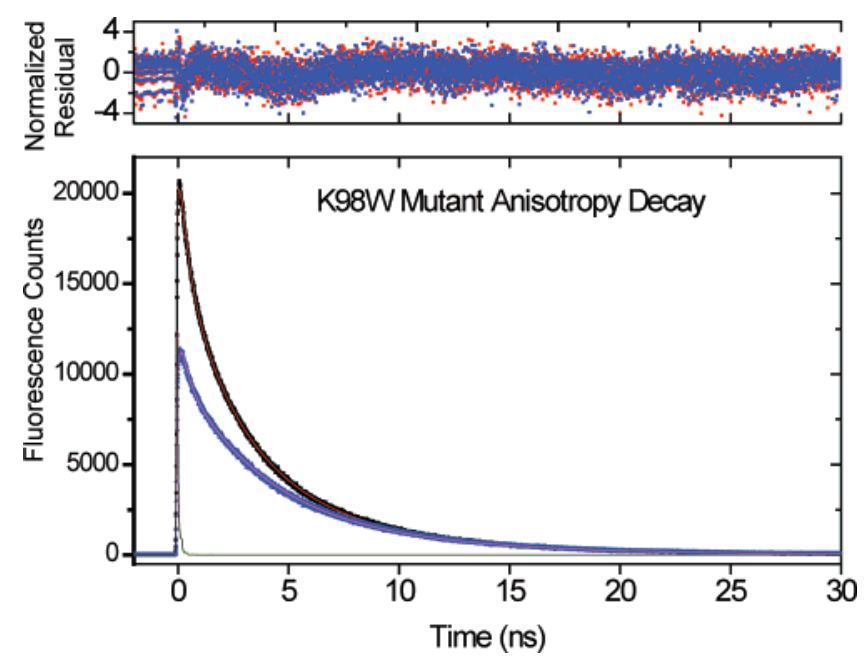

Figure 5. Time-resolved anisotropy fluorescence decay data for the Lys98Trp U1A protein (excitation at $305 \mathrm{~nm}$ and detection at $380 \mathrm{~nm}$ ). The lower traces are the raw parallel and perpendicular decay data overlaid with a solid line that is the best fit function. Also superimposed is the system response function. The top panel is the normalized residual between each fit and the raw data.

ized previously by Hall and co-workers, forming a data set to contrast with the data we have obtained. ${ }^{28}$ Substituting Trp into the relatively rigid $\beta$-sheet should eliminate any anisotropic decay associated with helix $\mathrm{C}$, while other decay components, such as molecular rotation of the protein, should be similar, although not necessarily identical, to those of the proteins labeled in helix $\mathrm{C}$.

During the analysis of the data from the Lys98Trp or Gly99trp proteins, distinguishing a two-component anisotropy fit from a three-component fit was initially troublesome. The $\chi^{2}$ values obtained from a three-component fit were typically not significantly better than the $\chi^{2}$ values for a two-component fit. However, the numbers obtained from the two-component fits did not make physical sense because the long component, which should be due to overall molecular rotation, was typically significantly shorter than previously measured ${ }^{28}$ and shorter than that expected based on simple hydrodynamic calculations. As an example, Table 4 shows a comparison of the anisotropy fitting parameters for the Phe56Trp and Lys98Trp U1A mutants and the double mutant Phe56Ala/Lys98Trp. A two-component fit for the Phe56Trp mutant, in which the Trp is located on the $\beta$-sheet, gives a reasonable two-component fit with a long component $(7.06 \mathrm{nsec})$ that is consistent with the published values $^{28}$ and the expectation from hydrodynamic estimates. ${ }^{44}$ A three-component fit converged with two components being the same, thus yielding similar values to the two-component fit. The mutants containing Trp at position 98 showed an unreasonably fast long component (4.51 nsec as shown in Table 4) when a two-component fit was used. A three-component fit yielded a slightly improved $\chi^{2}$ value and a more reasonable and consistent value for the long component of $\sim 7 \mathrm{~ns}$. The third anisotropy component was approximately $2 \mathrm{nsec}$ and is interpreted as the segmental motion of helix $\mathrm{C}$.

It might be argued for the Lys98Trp mutants that the $\sim 4 \mathrm{~ns}$ long component obtained with the two-component fit is due to a different orientation of the Trp with respect to the rotational axes of the molecule, as compared to Trp at the Phe56 position. However, the Gly99Trp U1A proteins in which the Trp probe in helix $\mathrm{C}$ is likely to be in a different orientation with respect to the rotational axes of the entire protein compared to Lys98Trp, exhibited the same behavior as the Lys98Trp U1A and gave similar three component anisotropy fits (Table 3). This supports 


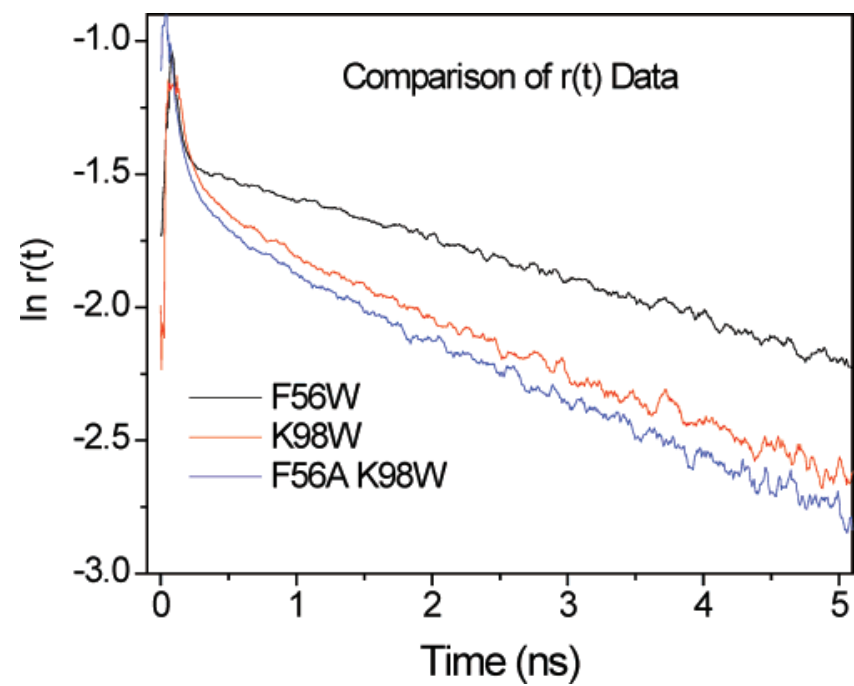

Figure 6. Natural log of the time-resolved anisotropy function, $r(t)$, for the $\mathrm{F} 56 \mathrm{~W}, \mathrm{~K} 98 \mathrm{~W}$, and F56AK98W U1A proteins. The $r(t)$ data was smoothed using a 10 point weighted average prior to taking the logarithm.

the proposal that the measured anisotropy behavior is characteristic of the helix motion and not just the local environment of the Lys98Trp species.

Direct inspection of the time-resolved anisotropy function, $r(t)$, is also useful for establishing the need for three anisotropy decay components. Figure 6 shows the natural $\log$ of $r(t)$ for the Phe56Trp, Lys98Trp, and Lys98Trp/Phe56Ala mutants. All three exhibit a fast component at early time and a substantial long time component that appears as a straight line in the log plot. The long component of the Phe56Trp mutant has a single, linear long time component which spans the entire time scale, except where it meets the fast time component at early time ( $\sim 0.2 \mathrm{nsec}$ ). The log plots for the Lys98Trp and the Phe56Ala/ Lys98Trp mutants exhibit a similar long time component, but also show significant curvature in the region between 0.2 and $1.5 \mathrm{nsec}$. Thus, the three-component fit is justified for the U1A proteins labeled in helix $\mathrm{C}$, with the intermediate component assigned to be the segmental motion of helix $\mathrm{C}$.

Estimating the Cone Angle of Helix C Motion. A simple model for hindered rotational motion in a cone was used to estimate the range of motion of helix $\mathrm{C}$ from the loss of anisotropy associated with its motion. Using the equation shown below and the correlation time of helix $\mathrm{C}$ motion determined for Lys98Trp, we estimated the cone angle of motion of helix $\mathrm{C}$ to be $20^{\circ}$.

$$
\begin{gathered}
\frac{r_{\infty}}{r_{0}}=\left\langle\frac{3 \cos ^{2} \psi-1}{2}\right\rangle^{2} \\
\frac{r_{\infty}}{r_{0}} \equiv \frac{\beta_{3}}{\beta_{2}+\beta_{3}} \equiv S^{2}=\text { order parameter }=0.68 \\
\psi=20^{\circ}
\end{gathered}
$$

As defined in eq 1, $r_{\infty}$ is the anisotropy at $t=\infty$ and $r_{0}$ is the anisotropy at $t=0$. On the basis of this cone angle, we determined that the motion of helix $\mathrm{C}$ observed on the nanosecond time scale in the time-resolved fluorescence anisotropy experiments did not represent a transition between the open and closed forms. A transition of helix $\mathrm{C}$ between the closed and open forms would correspond to a cone angle greater than $90^{\circ}$ and result in a complete loss of anisotropy. Similar results were found for the Gly99Trp U1A protein.

The estimates of the cone angle of helix $\mathrm{C}$ are consistent with the results of MD simulations on the wild-type protein reported previously. ${ }^{27,36}$ The MD simulations showed the helix moving not simply in an angular fashion but as a combination of an angular motion with a translation across the face of the $\beta$-sheet (Figure 7). An estimate of the angular part of this motion gave good agreement with our measured value of $20^{\circ}$.

An analysis of the solvent accessible surface area of Phe56, Lys98, and Gly99 from the MD simulation of the wild typeprotein was performed. The solvent accessibility of these residues compared to an interior residue, Leu30, and a solvent exposed residue, Ser91, over the last $3 \mathrm{~ns}$ of the simulation is shown in Figure 8. The SASA values were normalized by the extended Ala-X-Ala SASA values for each residue. These data show that Phe56 is sequestered from solvent compared to Lys98 and Gly99 and that Lys98 is nearly as solvent exposed as Ser91. This result is consistent with the blue-shifted emission wavelength of the Phe56Trp protein relative to the Lys98Trp and Gly99Trp proteins and with previously reported acrylamide quenching experiments, which showed that Phe56Trp is sequestered from solvent relative to other positions of Trp labeling. ${ }^{28}$ Thus, it is our view that the observed motion of helix $\mathrm{C}$ in these experiments is a fluctuation within the closed form.

Mutations of the U1A Protein. Three mutations were incorporated into the Lys98Trp U1A protein to probe the contributions of residues on the surface of the $\beta$-sheet and in the hinge region between helix $\mathrm{C}$ and the $\beta$-sheet to the dynamics of helix $\mathrm{C}$ and the stability of the complex (Figure 9). The first mutation examined was Phe56Ala. In the closed form of $\mathrm{U} 1 \mathrm{~A}$, helix $\mathrm{C}$ interacts with the surface of the $\beta$-sheet, forming a small hydrophobic core between Ile93, Ile94, Met97, Phe56, Leu44, and Ile58. ${ }^{10}$ Phe56 stacks with A6 in the loop of SL2 RNA in the complex. ${ }^{11}$ The substitution of Ala for Phe56 could change the interactions of helix $\mathrm{C}$ with the $\beta$-sheet, leading to altered helix $\mathrm{C}$ dynamics. The second position of mutation was Asp90, which is located in the hinge region between helix $\mathrm{C}$ and the $\beta$-sheet. This residue, along with Thr89 and Ser91, undergoes large rotations of $\Psi$ and $\Phi$ angles upon binding to RNA. ${ }^{10}$ The backbone carbonyl of Asp 90 hydrogen bonds to the exocyclic amine of C7 in SL2 RNA in the complex. ${ }^{11}$ Two mutations were introduced at this position: Asp90Ala and Asp90Gly. The Asp90Ala substitution replaces the charged side chain with a small, aliphatic residue, while the Asp90Gly substitution was expected to not only eliminate the side chain, but also add significant flexibility to the hinge region.

The overall structural integrity of the mutant U1A proteins and their ability to bind RNA was investigated using CD spectroscopy and gel shift assays, respectively. The CD spectra of the three mutants were similar to those of the Lys98Trp and wild type U1A proteins, suggesting these mutations do not cause large alterations of protein structure. The binding affinity of the Lys98Trp/Phe56Ala protein for SL2 RNA is very weak, which is consistent with that measured previously for the F56A protein. ${ }^{50}$ The complexes formed with the Asp90Ala/Lys98Trp and Asp90Gly/Lys98Trp proteins were destabilized by 1.5 and $3.1 \mathrm{kcal} / \mathrm{mol}$, respectively, compared to the complex formed with the Lys98Trp protein (Table 1).

The steady-state fluorescence spectra of the Phe56Ala, Asp90Ala, and Asp90Gly U1A proteins are shown in Figure 10. The emission maxima of these proteins are similar to that of the Lys98Trp protein. Interestingly, the emission maxima of the Phe56Ala and Asp90Gly protein shift to longer wavelength 


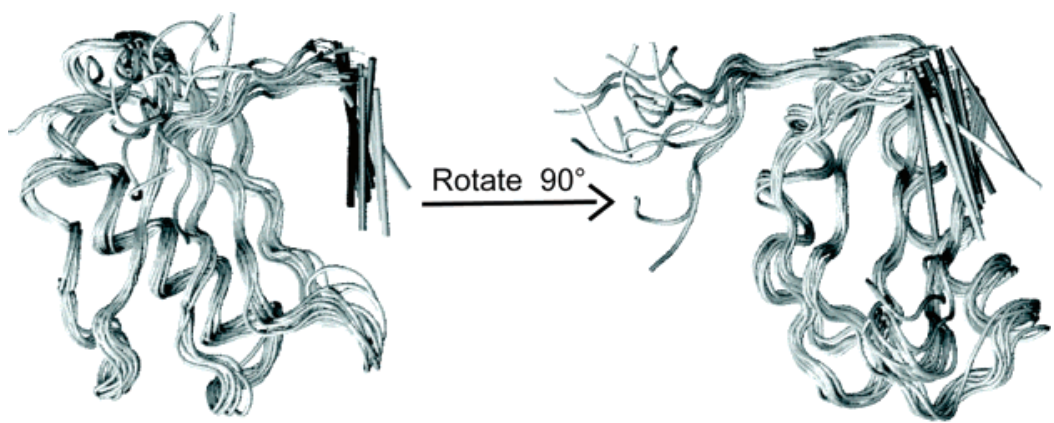

Figure 7. Overlay of structures obtained from the MD simulation of U1A protein. ${ }^{27,36}$ Snapshots were collected once every 500 ps over the 5 ns trajectory and oriented by superposition of the $\mathrm{C}-\alpha$ atoms of the $\beta \alpha \beta \beta \alpha \beta$ motif. Helix $\mathrm{C}$ is indicated by narrow rods that span the range of grayscale from white at $0 \mathrm{~ns}$ to black at $5 \mathrm{~ns}$ to track the motion of the helix over the course of the simulation.

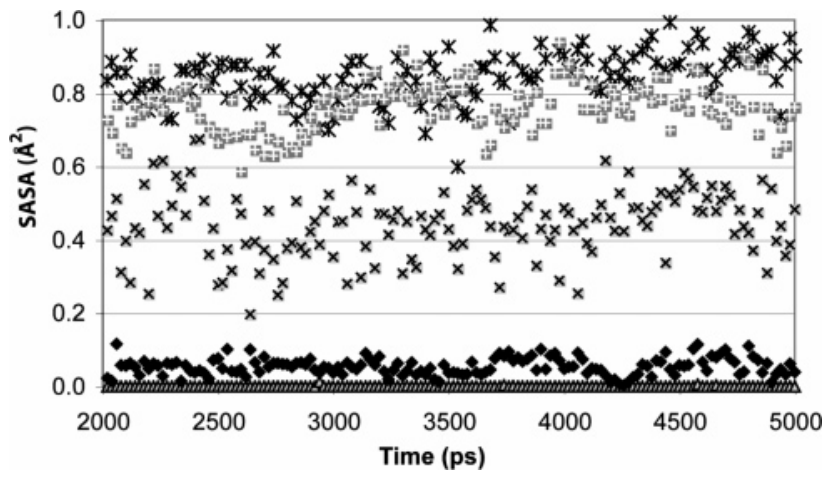

Figure 8. Solvent accessible surface area (SASA) of select residues in the wild-type U1A protein computed from MD simulations over the converged portion of the trajectory at 20 ps intervals: Leu30 $(\mathbf{\Delta})$, Phe56 $(\diamond)$, Ser91 (*), Lys98 (+), and Gly99(×). The SASA values were normalized by the extended Ala-X-Ala SASA values for each residue, $\mathrm{X}$.

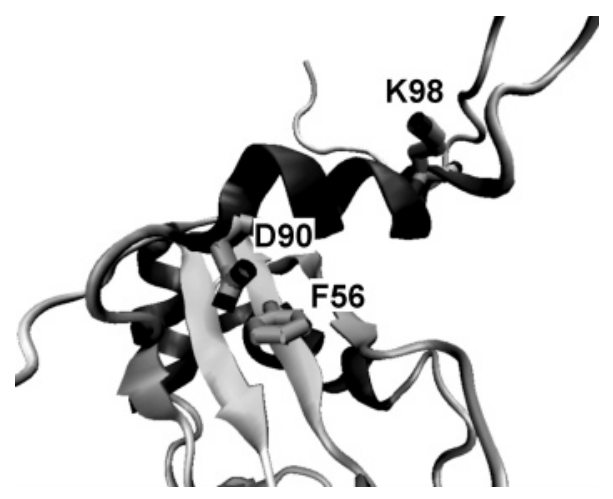

Figure 9. Diagram of the structure of the free U1A protein showing the positions of incorporation of Phe56Ala, Asp90Ala, and Asp90Gly mutations.

with increasing excitation wavelength, similar to the Phe56Trp protein. These data may indicate an alteration in the environment of Trp98 upon incorporation of these mutations into the protein.

The time-resolved decay data for these proteins are similar to those of Lys98Trp U1A protein discussed previously (Table $2)$. The observation that the fluorescence decay is not altered by the Phe56Ala or Asp90Gly mutations is similar to the results of an investigation of the effects of eliminating helix $\mathrm{C}$ on the fluorescence of Phe56Trp U1A. ${ }^{28}$ In this investigation, the steady-state fluorescence, but not the decay data, of Phe56Trp were altered by the deletion of helix $C$, suggesting a weak interaction between helix $\mathrm{C}$ and the surface of the $\beta$-sheet. Only the Asp90Gly mutant contains two values, that for $\alpha_{1}$ and $\tau_{3}$, that are outside of the experimental error of the measurements for the Lys98Trp protein.
The three components of the time-resolved fluorescence anisotropy decay of the Phe56Ala/Lys98Trp protein are within experimental error of those of the Lys98Trp protein. The values for some of the fractional contributions of the decay components and the time scale of the slowest component of the Asp90Ala/ Lys98Trp and Asp90Gly/Lys98Trp proteins differ from those of the Lys98Trp protein. Compared to the Lys98Trp protein, the Asp90Ala/Lys98Trp and Asp90Gly/Lys98Trp mutants have a greater fast component contribution $\left(\beta_{1}\right)$, a smaller middle component contribution for Asp90Ala $\left(\beta_{2}\right)$, and a faster, slow component $\left(\phi_{3}\right)$. The complexity of the dynamic response to mutations is not surprising because the ensemble of dynamic motions are not likely to all fall into narrow categories of fast, intermediate, and slow dynamics. The results of molecular dynamics simulations agree with the fluorescence anisotropy experiments and predict that the dynamics of helix $\mathrm{C}$ are similar in the wild-type and mutant proteins. ${ }^{36,51}$

\section{Discussion}

The data presented here suggest that helix $\mathrm{C}$ of the $\mathrm{N}$-terminal RRM of the U1A protein is dynamic in the closed conformation on a $2-3$ ns time scale with a limited range of motion estimated to be $20^{\circ}$. The position of labeling was chosen to be directed away from the surface of the $\beta$-sheet to isolate the dynamic contributions of helix $\mathrm{C}$ from complications in spectral interpretations due to interactions of the fluorophore with the surface of the $\beta$-sheet. Consistent with this design, the steady-state fluorescence measurements suggest that the Trp fluorophores in Lys98Trp and Gly99Trp are solvent exposed, and binding measurements indicate that these substitutions do not alter interactions with RNA. Comparison with the steady-state fluorescence spectrum of the Phe56Trp U1A protein suggests that while helix $\mathrm{C}$ is likely to be interacting with the surface of the $\beta$-sheet, and thus, altering the fluorescent properties of Phe56Trp, these interactions do not alter the spectral properties of Lys98Trp or Gly99Trp. A solvent accessibility analysis of the MD simulation suggests that Phe56 is sequestered from solvent even though helix $\mathrm{C}$ is dynamic. Together, these data indicate that helix $\mathrm{C}$ is dynamic within the closed conformation and is not exchanging between the closed and open forms on the nanosecond time scale.

These results contribute to a developing understanding of the dynamics of helix $\mathrm{C}$ in the U1A protein and the roles of these dynamic processes in complex formation. A previous investigation explored indirectly the dynamics of helix $\mathrm{C}$ with timeresolved fluorescence measurements of the Phe56Trp U1A mutant. ${ }^{28}$ Analysis of steady-state fluorescence data and acrylamide quenching experiments indicated that solvent exposure of Trp56 increased significantly upon truncation of helix C. The emission maximum shifted to longer wavelength as the excita- 

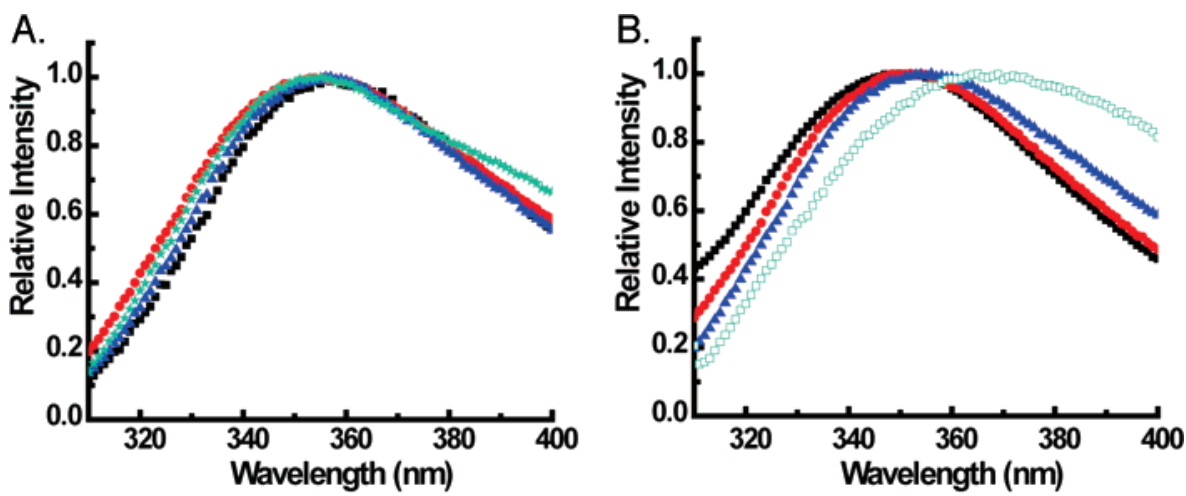

Figure 10. (A) Steady-state fluorescence spectra of Lys98Trp (blue), Asp90Ala/Lys98Trp (light blue), Asp90Gly/Lys98Trp (black), and Phe56Ala/ Lys98Trp (red) proteins $(1 \mu \mathrm{M})$ using a $\lambda_{\text {exc }}$ of $295 \mathrm{~nm}$ at $25^{\circ} \mathrm{C}$ in $50 \mathrm{mM} \mathrm{KCl}$ and $10 \mathrm{mM} \mathrm{K}_{2} \mathrm{HPO}_{4}(\mathrm{pH}$ 7.4). (B) Steady-state fluorescence spectra of Phe56Ala/Lys98Trp at $25^{\circ} \mathrm{C}$ in $50 \mathrm{mM} \mathrm{KCl}$ and $10 \mathrm{mM} \mathrm{K}_{2} \mathrm{PO}_{4}$ (pH 7.4) at different excitation wavelengths: $285 \mathrm{~nm}$ (black), $290 \mathrm{~nm}$ (red), 295 $\mathrm{nm}$ (blue), and $300 \mathrm{~nm}$ (light blue).

tion wavelength was increased for U1A(2-102), but not for the protein lacking helix C, U1A(2-93). The authors concluded from these observations that helix $\mathrm{C}$ is dynamic on the nanosecond or longer time scale. However, the deletion of helix $\mathrm{C}$ did not alter the time-resolved fluorescence parameters of Trp56 or the time-resolved anisotropy decay of the Phe56Trp protein, suggesting that the interaction between Trp56 and helix $\mathrm{C}$ is weak. NMR characterization of the dynamics of the U1A protein suggested that helix $\mathrm{C}$ is dynamic and, in particular, exhibits motions on the micro to millisecond time-scale in the U1A(2102) construct, ${ }^{29}$ which is a time scale not probed by timeresolved fluorescence anisotropy experiments. The results from the experiments described here define more directly the motions of helix $\mathrm{C}$ and show that helix $\mathrm{C}$ is dynamic, as suggested by previous experiments, but within a limited range of motion on the nanosecond time scale.

The observation that the Phe56Ala mutations do not alter the dynamics of helix C suggests that the greater than 100000-fold destabilization of the complex that results from the Phe56Ala mutation is not due to a change in the dynamics of helix $\mathrm{C}$ in the free protein on the picosecond-nanosecond time scale. Previous investigations of the kinetics of association and dissociation of the U1A-RNA complex have shown that the Phe56Ala mutation only affects the dissociation rate, but not the association rate, of the U1A-SL2 RNA complex. ${ }^{52}$ Thus, the destabilization of the complex upon mutation of Phe56 to Ala most likely results from changes in the energetics of the complex.

The small effect of the Asp90Ala or Asp90Gly mutations on the dynamics of helix $\mathrm{C}$ is consistent with helix $\mathrm{C}$ undergoing limited dynamical motions on the nanosecond time scale instead of equilibrating between open and closed conformations that would require large conformational changes of Asp90. Although both mutations destabilize the complex, as expected, the Gly mutation destabilizes the complex more than the Ala mutation. Both mutations may alter the flexibility of the hinge region, thus changing the energetics of the conformational change required upon binding, or the hydrogen bond formed between the backbone carbonyl of Asp90 with C7 in SL2 RNA. These substitutions may also alter local dynamics in the hinge region and thus weaken the interactions of adjacent amino acids (Ser91 and Thr89) with the RNA target.

\section{Conclusions}

Short time-scale dynamic processes, like those identified here for helix $\mathrm{C}$, have been shown to have important roles in ligand binding $^{53,54}$ and enzyme catalysis ${ }^{55-57}$ and have been suggested to contribute to longer time-scale dynamic processes on the micro- and millisecond time scale. ${ }^{58-61}$ Thus, the dynamics of helix $\mathrm{C}$ investigated here may contribute to the conformational change away from the surface of the $\beta$-sheet required upon binding, even though the time scale of this conformational change may be longer than is detected by time-resolved anisotropy experiments. In addition, a relationship has been implicated between dynamic processes on the pico- to nanosecond time scale and the propagation of the long-range signals required for cooperative interactions. ${ }^{34,35,62-64}$ Because conformational changes in $\mathrm{C}$-terminal helices upon complex formation are common in the formation of RRM-RNA complexes, the variation of the dynamics of this region of RRMs may be an important mechanism for gaining affinity or specificity for RNA targets.

Acknowledgment. Funding was provided by the NIH (Grant GM-56857 (A.M.B.) and Grant GM-076490 (D.L.B.)). B.L.K. was supported by a NIH Postdoctoral Fellowship (F32GM072345). M.A. was supported by awards for undergraduate research from the Howard Hughes Medical institute.

\section{References and Notes}

(1) Rubin, G. M.; Yandell, M. D.; Wortman, J. R.; Miklos, G. L. G.; Nelson, C. R.; Hariharan, I. K.; Fortini, M. E.; Li, P. W.; Apweiler, R.; Fleischmann, W.; Cherry, J. M.; Henikoff, S.; Skupski, M. P.; Misra, S.; Ashburner, M.; Birney, E.; Boguski, M. S.; Brody, T.; Brokstein, P.; Celniker, S. E.; Chervitz, S. A.; Coates, D.; Cravchik, A.; Gabrielian, A.; Galle, R. F.; Gelbart, W. M.; George, R. A.; Goldstein, L. S. B.; Gong, F.; Guan, P.; Harris, N. L.; Hay, B. A.; Hoskins, R. A.; Li, J.; Li, Z.; Hynes, R. O.; Jones, S. J. M.; Kuehl, P. M.; Lemaitre, B.; Litleton, J. T.; Morrison, D. K.; Mungall, C.; O'Farrell, P. H.; Pickeral, O. K.; Shue, C.; Vosshall, L. B.; Zhang, J.; Zhao, Q.; Zheng, X. H.; Zhong, F.; Zhong, W.; Gibbs, R.; Venter, J. C.; Adams, M. D.; Lewis, S. Science 2000, 287, 2204.

(2) Lorkovic, Z. J.; Barta, A. Nucleic Acids Res. 2002, 30, 623.

(3) Birney, E.; Kumar, S.; Krainer, A. R. Nucleic Acids Res. 1993, 21, 5803.

(4) Maris, C.; Dominguez, C.; Allain, F. H.-T. FEBS J. 2005, 272, 2118.

(5) Krecic, A. M.; Swanson, M. S. Curr. Opin. Cell Biol. 1999, 11, 363.

(6) Varani, G.; Nagai, K. Annu. Rev. Biophys. Biomol. Struct. 1998, 27,407 .

(7) Burd, C. G.; Dreyfuss, G. Science 1994, 265, 615.

(8) Williamson, J. R. Nat. Struct. Biol. 2000, 7, 834

(9) Leulliot, N.; Varani, G. Biochemistry 2001, 40, 7947.

(10) Avis, J. M.; Allain, F. H.-T.; Howe, P. W. A.; Varani, G.; Nagai, K.; Neuhaus, D. J. Mol. Biol. 1996, 257, 398

(11) Oubridge, C.; Ito, N.; Evans, P. R.; Teo, C. H.; Nagai, K. Nature 1994, 372, 432.

(12) Perez-Canadillas, J. M.; Varani, G. EMBO J. 2003, 22, 2821.

(13) Deka, P.; Rajan, P. K.; Perez-Canadillas, J. M.; Varani, G. J. Mol. Biol. 2005, 347, 719. 
(14) Crowder, S. M.; Kanaar, R.; Rio, D. C.; Alber, T. Proc. Natl. Acad. Sci. 1999, 96, 4892.

(15) Handa, N.; Nureki, O.; Kuimoto, K.; Kim, I.; Sakamoto, H.; Shimura, Y.; Muto, Y.; Yokoyama, S. Nature 1999, 398, 579.

(16) Wang, X.; Tanaka-Hall, T. M. Nat. Struct. Biol. 2001, 8, 141.

(17) Deo, R. C.; Bonanno, J. B.; Sonenberg, N.; Burley, S. K. Cell 1999, 98,835 .

(18) Allain, R. H. T.; Bouvet, P.; Dieckmann, T.; Feigon, J. EMBO J. 2000, 19, 6870

(19) Stark, H.; Dube, P.; Lührmann, R.; Kastner, B. Nature 2001, 409 , 539.

(20) Green, M. R. Annu. Rev. Cell Biol. 1991, 7, 559.

(21) Hall, K. B. Biochemistry 1994, 33, 10076

(22) Boelens, W. C.; Jansen, E. J. R.; van Venrooij, W. J.; Stripecke, R.; Mattaj, I. W.; Gunderson, S. I. Cell 1993, 72, 881.

(23) Tsai, D. E.; Harper, D. S.; Keene, J. D. Nucl. Acids Res. 1991, 19 4931.

(24) Lu, J.; Hall, K. B. J. Mol. Biol. 1995, 247, 739

(25) Scherly, D.; Boelens, W.; van Venrooij, W. J.; Dathan, N. A.; Hamm, J.; Mattaj, I. W. EMBO J. 1989, 8, 4163.

(26) Rupert, P. B.; Xiao, H.; Ferré-D’Amaré, A. R. Acta Crystallogr. D 2003, D59, 1521

(27) Pitici, F.; Beveridge, D. L.; Baranger, A. M. Biopolymers 2002, $65,424$.

(28) Jean, J. M.; Clerte, C.; Hall, K. B. Protein Sci. 1999, 8, 2110.

(29) Mittermaier, A.; Varani, L.; Muhandiram, D. R.; Kay, L. E.; Varani,

G. J. Mol. Biol. 1999, 294, 967.

(30) Showalter, S. A.; Hall, K. B. J. Mol. Biol. 2004, 335, 465.

(31) Zeng, Q.; Hall, K. B. RNA 1997, 3, 303.

(32) Kranz, J. K.; Hall, K. B. J. Mol. Biol. 1998, 275, 465.

(33) Showalter, S. A.; Hall, K. B. J. Mol. Biol. 2002, 322, 533.

(34) Kormos, B. L.; Baranger, A. M.; Beveridge, D. L. J. Struct. Biol.

$\mathbf{2 0 0 7}, 157,500$

(35) Kormos, B. L.; Baranger, A. M.; Beveridge, D. L. J. Am. Chem.

Soc. 2006, 128, 8992

(36) Kormos, B. L.; Benitex, Y.; Baranger, A. M.; Beveridge, D. L. J.

Mol. Biol. 2007, 371, 1405

(37) Nagai, K.; Oubridge, C.; Jessen, T. H.; Li, J.; Evans, P. R. Nature $1990,348,515$

(38) Kunkel, T. A.; Bebenek, K.; McClary, J. Methods Enzymol. 1991, $204,125$.

(39) Shiels, J. C.; Tuite, J. B.; Nolan, S. J.; Baranger, A. M. Nucleic Acids Res. 2002, 30, 550 .

(40) Beechem, J. M.; Gratton, E. Fluorescence Spectroscopy Data Analysis Environment A Second Generation Global Analysis Program. In Time-Resolved Laser Spectroscopy in Biochemistry; Lakowicz, J., Ed.; SPIE: Bellingham, WA, 1988; Vol. 909, p 77.

(41) Case, D. A.; Darden, T. A.; Cheatham, T. E.; Simmerling, C. L.; Wang, J.; Duke, R. E.; Luo, R.; Merz, K. M.; Wang, B.; Pearlman, D. A.;
Crowley, M.; Brozell, S.; Tsui, V.; Gohlke, H.; Mongan, J.; Hornak, V.; Cui, G.; Beroza, P.; Schafmeister, C.; Caldwell, J. W.; Ross, W. S.; Kollman, P. A. Amber 8; University of California: San Francisco, CA, 2004

(42) Pearlman, D. A.; Case, D. A.; Caldwell, J. W.; Ross, W. S. Cheatham, T. E., III; DeBolt, S.; Ferguson, D.; Seibel, G.; Kollman, P. Comput. Phys. Commun. 1995, 92, 1.

(43) Ahmad, S.; Gromiha, M.; Fawareh, H.; Sarai, A. BMC Bioinformatics 2004, 5, 51 .

(44) Lakowicz, J. R. Principles of Fluorescence Spectroscopy, 2nd ed.; Kluwer Academic/Plenum: New York, 1999.

(45) Bailey, M. F.; Thompson, E. H. Z.; Millar, D. P. Methods 2001 25,62 .

(46) Deprez, E.; Tauc, P.; Leh, H.; Mouscadet, J.-F.; Auclair, C.; Hawkins, M. E.; Brochon, J.-C. Proc. Natl. Acad. Sci. U.S.A. 2001, 98 10090 .

(47) Kim, S.-J.; Beard, W. A.; Harvey, J.; Shock, D. D.; Knutson, J. R.; Wilson, S. H. 1. J. Biol. Chem. 2003, 278, 5072.

(48) Wahl, P.; Paoletti, J.; Le Pecq, J.-B. Proc. Natl. Acad. Sci. U.S.A 1970, 65, 417.

(49) Munro, I.; Pecht, I.; Stryer, L. Proc. Natl. Acad. Sci. U.S.A. 1979, 76,56 .

(50) Zhao, Y.; Baranger, A. M. J. Am. Chem. Soc. 2003, 125, 2480.

(51) Kormos, B. L.; Beveridge, D. L.; Baranger, A. M., unpublished results.

(52) Katsamba, P. S.; Myszka, D. G.; Laird-Offringa, I. A. J. Biol. Chem 2001, 276, 21476 .

(53) Fuentes, E. J.; Der, C. J.; Lee, A. L. J. Mol. Biol. 2004, 335, 1105. (54) Bracken, C.; Carr, P. A.; Cavanagh, J.; Palmer, A. G. J. Mol. Biol. 1999, 285, 2133 .

(55) Agarwal, P. K.; Billeter, S. R.; Rajagopalan, P. T. R.; Benkovic, S. J.; Hammes-Schiffer, S. Proc. Natl. Acad. Sci. U.S.A. 2002, 99, 2794 (56) Wong, K. F.; Selzer, T.; Benkovic, S. J.; Hammes-Schiffer, S. Proc Natl. Acad. Sci. U.S.A. 2005, 102, 6807.

(57) Liang, Z.-X.; Tsigos, I.; Bouriotis, V.; Klinman, J. P. J. Am. Chem. Soc. 2004, 126, 9500 .

(58) Eisenmesser, E. Z.; Bosco, D. A.; Akke, M.; Kern, D. Science 2002 295,1520

(59) Eisenmesser, E. Z.; Millet, O.; Labeikovsky, W.; Korzhnev, D. M Wolf-Watz, M.; Bosco, D. A.; Skalicky, J. J.; Kay, L. E.; Kern, D. Nature 2005, 438, 117

(60) Kalodimos, C. G.; Biris, N.; Bonvin, A. M. J. J.; Levandoski, M. M.; Guennuegues, M.; Boelens, R.; Kaptein, R. Science 2004, 305, 386

(61) Kovrigin, E. L.; Loria, J. P. Biochemistry 2006, 45, 2636.

(62) Lockless, S. W.; Ranganathan, R. Science 1999, 286, 295.

(63) Süel, G. M.; Lockless, S. W.; Wall, M. A.; Ranganathan, R. Nat. Struct. Biol. 2003, 10, 59 .

(64) Showalter, S. A.; Hall, K. B. Biophys. J. 2005, 89, 2046. 